\title{
HoxB4 : un déterminant crucial du potentiel de reconstitution de I'hématopoïèse définitive dans les cellules embryonnaires
}

Françoise Sainteny

$>$ L'hématopoïèse qui se développe dans les corps embryonnaires ( $\varepsilon B$ ) dérivés des cellules souches embryonnaires (cellules દS) de souris récapitule l'hématopoïèse embryonnaire du sac vitellin. Comme les cellules souches du sac vitellin, les cellules hématopoïétiques dérivées des cellules \&S ont longtemps été considérées comme incapables de reconstituer à long terme le tissu hématopoïétique d'une souris irradiée, incompétence attribuée soit à l'immaturité de ces cellules, soit à des anomalies dans leur adressage à la moelle osseuse $[1,2]$. Les tentatives ont toujours donné lieu à des reconstitutions lymphoïdes et/ou myéloïdes partielles et éphémères [1-4]. En aucun cas, une reconstitution hématopoïétique de receveurs secondaires par la moelle des receveurs primaires n'a été observée. Cela signe l'absence, ou la faible concentration, dans la moelle primaire, de cellules primitives répondant aux critères de définition de la cellule souche hématopoïétique (CSH), autorenouvellement et multipotentialité.

Les mécanismes moléculaires contrôlant le passage de l'hématopoï̀se primitive à I'hématopoïèse définitive sont très mal connus. On sait cependant que plusieurs homéogènes sont exprimés dans les CSH définitives, à l'inverse de celles du sac vitellin. II s'agit de HoxB3, B4, A4 et $A 5$ [5]. Ainsi, HoxB4 apparaissait comme un bon candidat pour promouvoir l'hématopoïèse définitive. On savait par ailleurs que : (1) l'introduction d'HoxB4 dans la moelle osseuse adulte induit une stimulation de la capacité de repopulation de cette dernière sans interférer avec la différenciation [6] ; (2) HoxB4 est impliqué dans l'autorenouvellement des CSH définitives [6]; (3) HoxB4 stimule la production de colonies hématopoiétiques mixtes (contenant des cellules de plusieurs lignées) à partir des cellules ES [7]. Une étude parue au mois d'avril 2002 dans la revue Cell montre que l'expression ectopique du gène HoxB4 dans les cellules ES, associée à leur co-culture avec une lignée stromale, induit la commutation de l'hématopoïèse primitive dérivée de cellules $\varepsilon S$ à l'hématopoïèse définitive et permet la reconstitution lympho-myéloïde à long terme de receveurs primaires et secondaires [8]. Les cellules du sac vitellin acquièrent les mêmes propriétés en subissant le même traitement. L'expression d'HoxB4 dans les cellules de sacs vitellins prélevés avant la mise en circulation du sang (stade $\varepsilon 8,25$ ) est obtenue à l'aide d'un rétrovirus co-exprimant la GFP, protéine fluorescente qui permet la sélection des cellules infectées, puis leur identification et leur tri dans la moelle des souris receveuses. Une phase de culture des cellules transduites avec la lignée stromale murine 0p9, qui permet le développement des progéniteurs hématopoïétiques dérivés des cellules ES [9] est introduite dans le protocole avant injection à des souris adultes irradiées létalement. Ces receveurs présentent une reconstitution myélo-lymphoïde de type donneur $\left(G^{+}\right)$importante : $15,34,26$ et $5 \%$ de cellules de moelle sont respectivement $\mathrm{Grl}^{+}$(granuleux), Mac-l' ${ }^{+}$(macrophages),
Inserm U.362, Institut

Gustave Roussy, 39, rue

Camille Desmoulins, 94800

Villejuif, France.

$\mathrm{B}^{2} 20^{+}$(lymphocytes B) et $\mathrm{CD} 8^{+}$(lymphocytes $T$ ). La moelle de ces receveurs primaires est capable de reconstituer des receveurs secondaires jusqu'à cinq mois au moins après la greffe : à ce stade, le sang périphérique de ces derniers contient, parmi les cellules dérivées de la moelle primaire $\left(\mathrm{GFP}^{+}\right), 80 \%$ de cellules positives pour GR-1, $8 \%$ pour B220 et $9 \%$ pour CD 4 ou CD8. L'expression d'HoxB4 dans les précurseurs primitifs du sac vitellin induit donc un potentiel de CSH définitives.

L'expression d'HoxB4 au cours de la différenciation des cellules $\varepsilon S$ a été réalisée grâce à l'introduction dans les cellules $E S$ d'un transgène dans lequel la séquence d'HoxB4 est placée sous le contrôle d'un promoteur inductible par la doxycycline (Figure 1). L'expression d'HoxB4 est induite au $4^{e}$ jour de différenciation des cellules $\varepsilon S$ en corps embryonnaires et ceci pendant 48 heures. Cette période de différenciation a été choisie car elle correspond probablement à l'étape au cours de laquelle l'hémangioblaste dérivé des cellules ES $(\rightarrow)$ s'engage dans la différenciation vers la CSH primitive. La culture en méthylcellulose $(\rightarrow) \mathrm{m} / \mathrm{s}$ des cellules d'EB à l'issue de $1999, \mathbf{n}^{\circ} \mathbf{2}$, la période de surexpression p. 292 du gène HoxB4 montre que celle-ci induit une stimulation très marquée des progéniteurs les plus primitifs détectables par ce test, les CFU-GEMM (colony-forming unit granulocyte-erythroid-macrophage- 
megakaryocyte), alors que les (FU-GEMM produites par les cellules d'EB non transfectées sont rares et ont un potentiel érythroïde très limité. Les colonies dérivées des EB exprimant HoxB4 sont, comme celles de la moelle, de plus grande taille, très denses et majoritairement érythroïdes. Les cellules d'EBs sont ensuite cultivées sur la lignée $0 p 9$ en présence de cytokines et en maintenant l'induction d'HoxB4 par la doxycycline. Des colonies semi-adhérentes de type blastique se développent, majoritairement composées de cellules exprimant des marqueurs caractéristiques de la CSH définitive, c-kit ( $81 \%$ des cellules) et CD31

(78\%). Une plus faible proportion de cellules exprime les marqueurs de différenciation macrophagique (Mac-1 : $21 \%$ ) et granulocytaire (Gr-1 : $6 \%$ ). Les marqueurs érythroïde (Terl19) et lymphocytaire (B220) sont encore plus minoritaires $(0,7$ et $0,6 \%)$. La culture des cellules surexprimant HoxB4 aboutit donc à la formation d'une population de CSH primitives qui semblent s'autorenouveler mais se différencier. Cette population, peu analysée par RT-PCR, exprime la globine $\beta$ majeure, suggérant que l'expression d'HoxB4 et la lignée Op9 éteignent l'érythropoïèse embryonnaire. Un autre élément important est l'activation de l'expression du récepteur de la chimiokine SDF-1, CXCR-4, dont la présence est requise pour l'adressage des cellules souches à la moelle osseuse $[9,10]$ et celle du gène Tel, impliquée dans la transition de l'hématopoïèse du foie fotal à celle de la moelle osseuse [11] prouvant que l'expression d'HoxB4, combinée à une phase d'expansion cellulaire sur la lignée $0 p 9$, confère l'apparition des marqueurs de l'hématopoï̀se définitive à des cellules dérivées de cellules embryonnaires. La capacité des cellules $\varepsilon S$ de reconstituer l'hématopoïèse à long terme, prouvant la présence de CSH primitives, est clairement montrée. Cinq à $32 \%$ de cellules d'origine du greffon $\left(\mathrm{GFP}^{+}\right)$sont retrouvées dans la moelle des receveurs irradiés 2 semaines après la greffe. Quinze semaines plus tard, une reconstitution substantielle de tous les lignages est observée : reconstitution myéloïde $\mathrm{GR}-\mathrm{I}^{+}$
(71 \%), Mac-1, et lymphoïde (B220+ : $30 \%, \mathrm{CD}^{+}: 22 \%$ et $\mathrm{CD} 8^{+}: 5 \%$ ). Les marqueurs des CSH, c-kit, Sca-l et AA4- 1 sont aussi détectés sur les cellules $\mathrm{GFP}^{+}$de la moelle des receveurs. L'induction d'HoxB4 in vivo n'est pas nécessaire pour obtenir la prise de greffe, suggérant que l'expression d'HoxB4 pendant la phase de culture suffit à conférer le potentiel de reconstitution de l'hématopoïèse définitive aux cellules dérivées des cellules ES. Ces cellules ont la capacité de reconstituer des receveurs secondaires pendant au moins 5 mois, montrant sans aucune ambiguïté que l'induction transitoire d'HoxB4 dans

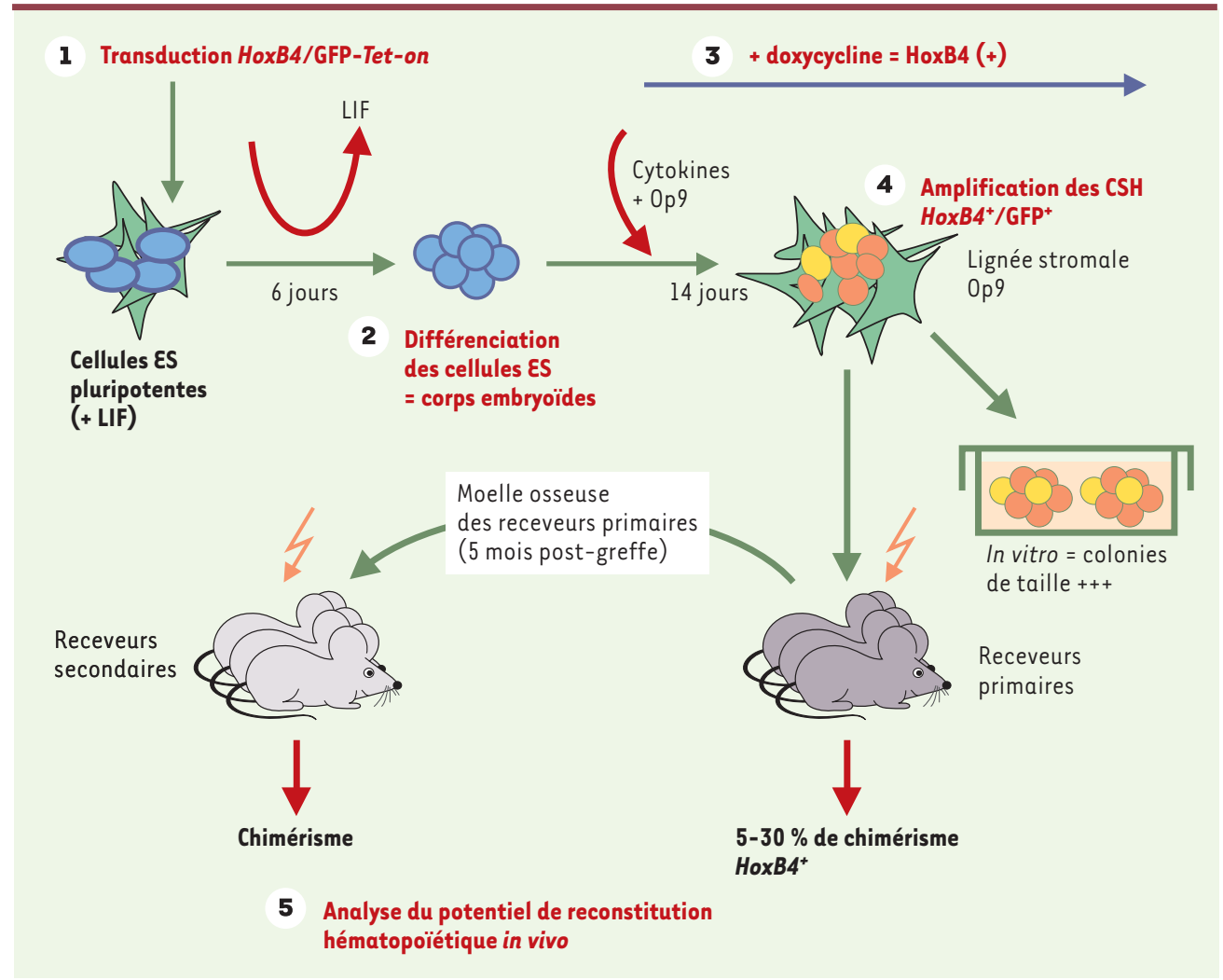

Figure 1. Induction d'un potentiel de cellule souche hématopöétique définitive dans les cellules ES. Les cellules $\varepsilon S$, maintenues à l'état pluripotent en présence d'une source de LIF (leukemia inhibiting factor), sont transduites avec un rétrovirus codant pour HoxB4 sous contrôle d'un promoteur inductible par la doxocycline. Les cellules $\varepsilon S$ sont sevrées de LIF, ce qui entraîne leur différenciation en corps embryoïdes. Ceux-ci sont exposés 48 heures à la doxocycline pour induire HoxB4 et transférés pendant 14 jours en présence de cytokines et de la lignée stromale 0p9. L'ajout de doxocycline est prolongé. Le potentiel des cellules obtenues après cette phase d'amplification cellulaire est analysé in vitro (test de colonies) et in vivo dans un système de transplantation hématopoïétique à des receveurs irradiés. La moelle et le sang des receveurs sont analysés pour la présence de cellules d'origine de donneur, et la moelle est injectée à des receveurs secondaires irradiés afin de tester la présence, chez le receveur primaire, de cellules souches hématopoïétiques. II faut noter que l'ajout de doxocycline n'est pas maintenu in vivo, mais est limité aux 14 jours de culture in vitro. 
les cellules dérivées des cellules ES a permis l'émergence d'une CSH dotée de la potentialité de reconstituer l'hématopoï̀se adulte. On ne sait pas aujourd'hui si HoxB4 détermine l'acquisition de cette potentialité chez l'embryon. De même, compte tenu de la redondance des gènes, il est possible que d'autres gènes de la famille Hox, notamment HoxA4, soient capables de provoquer l'émergence de la CSH définitive. Néanmoins, les résultats convaincants que décrit cet article sont de première importance dans le $\rightarrow$ m/s contexte de l'aptitude des

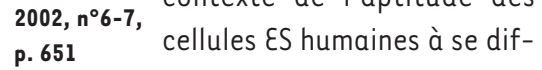
férencier en cellules hématopoïétiques [12] et de l'intérêt croissant pour la thérapie cellulaire en médecine réparatrice. II faut enfin signaler que ce modèle a permis la correction d'une anomalie génétique de la souris en combinant transplantation nucléaire, thérapie génique et cellulaire [13] $(\rightarrow)$. $\diamond$ HOXB4 triggers long term reconstituting capability to \&S cells and yolk sac-derived cells

\section{RéFÉRENCES}

1. Muller A, Dzierzak EA. દS cells have only a limited potential after adoptive transfer into mouse recipients. Development 1993 ; 118 : 1343-51.

2. Yoder MC, Hiatt K. Engraftment of embryonic hematopoietic cells in conditioned newborn recipients. Blood 1997 ; 89: 2176-83.

3. Potocnik AJ, Kohler H, Eichman K. Hematolymphoid in vivo reconstitution potential of subpopulations derived from in vitro differentiated embryonic stem cells. Proc Natl Acad Sci USA.

4. Mitjavila MT, Filippi MD, Cohen-Solal K, et al. The $\mathrm{Mpl}$-ligand is involved in the growth-promoting activity of the murine stromal cell line MS-5 on ES cell-derived hematopoiesis. Exp Hematol 1998 ; 26 : 124-34.
5. Sauvageau G, Lansdorp PM, Eaves CJ, et al. Differential expression of homeobox genes in functionally distinct CD34+ subpopulations of human bone marrow cells. Proc Natl Acad Sci USA 1994 ; 91 : 12223-7.

6. Sauvageau G, Thorsteinsdottir U, Eaves CJ, et al. Overexpression of HOXB4 in hematopoietic cells causes the selective expansion of more primitive populations in vitro and in vivo. Genes Dev 1995 ; 9 : 1753-65.

7. Helgason CD, Sauvageau G, Lawrence HJ, Largman C, Humpries RK.

Overexpression of HOXB4 enhances the hematopoietic potential of embryonic stem cells differentiated in vitro. Blood 1996 ; 87 : 2740-9.

8. Kyba M, Perlingeiro RCR, Daley GQ. HoxB4 confers definitive lymphoid-myeloid engraftment potential on embryonic stem cell and yolk sac hematopoietic progenitors. Cell $2002 ; 109$ : 29-37.

9. Nakano T, Kodama H, Honjo T. Generation of

lymphohematopoietic cells from embryonic stem cells in culture. Science 1994 ; 265 : 1098-101.

10. Peled A, Petit I, Kollet 0 , et al. Dependance of human stem cell engraftment and repopulation NOD/SCID mice on CXCR4. Science 1999 ; $283: 845-7$.

11. Wang LC, Swat W, Fujiwara $Y$, et al. The TEL/ETV6 gene is required specifically for hematopoiesis in the bone marrow. Genes Dev 1998 ; 12 : 2392-402.

12. Kaufman DS, Hanson ET, Lewis RL, Auerbach R, Thomson JA. Hematopoietic colony-forming cells derived from human embryonic stem cells. Proc Natl Acad Sci USA 2001 ; 98 : 10716-21.

13. Rideout WM, 3rd, Hochedlinger K, Kyba M, Daley GQ, Jaenisch R. Correction of a genetic defect by nuclear transplantation and combined cell and gene therapy. Cell 2002; 109 : 17-27.

\section{NOUVELLE}

\section{«Clonage thérapeutique »: de la théorie à la pratique}

Laure Coulombel

Inserm U.421,

Faculté de médecine,

8, rue du Général Sarrail,

94010 Créteil, France.
BCR. II en résulte un profond déficit immunitaire. La lignée NK (natural killer), dont la différenciation ne comprend
> L'article publié par le groupe de R. Jaenish dans Cell [1] est remarquable à plus d'un titre : c'est le premier essai expérimental grandeur nature de réparation génétique chez la souris à partir de cellules ES (embryonic stem cells) obtenues par transfert de noyaux somatiques. Succès oui, mais partiel en raison de la survenue de problèmes inattendus... Ce qui frappe aussi à la lecture de cet article, c'est que le travail tire parti de toutes les technologies de manipulation du génome développées au cours de ces
10 dernières années, recombinaison homologue dans les cellules $E S$, excision génétique par le système Cre/lox, transfert nucléaire, et, à ce titre, montre l'ampleur du chemin parcouru et la mâ̂trise acquise.

Les auteurs ont choisi de « réparer »le déficit immunitaire des souris Rag2 $^{-/-}$. Ces animaux, dont la mutation a été créée par recombinaison homologue, sont dépourvus de la recombinase permettant le réarrangement des gènes d'immunoglobulines formant les récepteurs TCR et aucune étape de réarrangement génique, est épargnée, ce qui a son importance pour la suite de l'histoire [2].

Première étape, obtenir une lignée de cellules ES « autologue » à partir d'une cellule somatique de ces souris par transfert nucléaire [3, 4] (Figure 1). Les noyaux de cellules de souris Rag2 $^{-/-}$ mâles ont été transférés dans des ovocytes énucléés bloqués au stade de métaphase II (202 ovocytes injectés), et ces cellules ont progressé jusqu'au stade de blastocystes « artificiellement » 